\title{
Kernos
}

Revue internationale et pluridisciplinaire de religion grecque antique

$31 \mid 2018$

Varia

\section{Cult Material. From Archaeological Deposits to Interpretation of Early Greek Religion}

\section{Graham Cuvelier}

\section{Q OpenEdition \\ Journals}

Édition électronique

URL : http://journals.openedition.org/kernos/2854

DOI : 10.4000/kernos.2854

ISSN : 2034-7871

Éditeur

Centre international d'étude de la religion grecque antique

Édition imprimée

Date de publication : 1 décembre 2018

Pagination : 318-320

ISBN : 978-2-87562-055-2

ISSN : 0776-3824

Référence électronique

Graham Cuvelier, «Cult Material. From Archaeological Deposits to Interpretation of Early Greek Religion », Kernos [En ligne], 31 | 2018, mis en ligne le 01 octobre 2018, consulté le 25 janvier 2021. URL : http://journals.openedition.org/kernos/2854; DOI : https://doi.org/10.4000/kernos.2854

Ce document a été généré automatiquement le 25 janvier 2021.

Kernos 


\title{
Cult Material. From Archaeological Deposits to Interpretation of Early Greek Religion
}

\author{
Graham Cuvelier
}

\section{RÉFÉRENCE}

Petra PAKKANEN, Susanne Bocher (éd.), Cult Material. From Archaeological Deposits to Interpretation of Early Greek Religion, Helsinki, 2015.1 vol. 17,5 × $25 \mathrm{~cm}, 155$ p. +11 pl. (Papers and Monographs of the Finnish Institute at Athens, 21). ISBN : 978-952-672119-4.

1 Ce volume contient les actes des journées d'étude intitulées « Defining and Interpreting Ancient Greek Cult Deposits ", organisées par le Finnish Institute at Athens et par le Deutsches Archäologisches Institut Athen, en octobre 2008, sur le site archéologique d'Olympie. L'objectif de cette rencontre internationale, réunissant quatorze chercheurs, était de réfléchir à des outils d'analyse et d'interprétation - tant théoriques que pratiques - des dépôts votifs en les rapportant plus intimement à leur contexte archéologique, particulièrement dans le cas des périodes pour lesquelles les sources écrites contemporaines font défaut en ce qui concerne les pratiques cultuelles. Le programme de ces trois journées d'étude comptait onze interventions. Les actes qui en découlent publient sept d'entre elles. Il s'agit de celles qui ont suscité les commentaires les plus pertinents et qui ont ainsi donné lieu aux débats les plus intéressants. Ces discussions sont résumées dans l'introduction (p. 3-7) rédigée par les deux éditrices de ce recueil d'articles.

2 L'ouvrage est ensuite divisé en deux sections. La première, qui porte le sous-titre « Cult and Material: Tradition, Theory and Materiality", est davantage théorique. Elle regroupe trois articles qui s'attèlent à définir ce qu'est un dépôt votif, notamment en portant un regard critique et méthodologique sur les recherches passées concernant 
cette problématique. - Dans « Worshiping Archaeologies - Theoretical Landscape in the Archaeological Study of Greek Religion and Cult Deposit» (p. 9-24), J. Siapkas présente un historique des différentes traditions propres à l'archéologie classique. Il dégage alors quatre approches (archéologique "pure», historique, artistique et anthropologique) et met en parallèle leurs développements respectifs au cours du $\mathrm{xx}^{\mathrm{e}}$ siècle. Dans un second temps, souhaitant dépasser cette catégorisation, l'A. propose les prémices d'un modèle conceptuel global qui tient compte de ces différentes approches en les réunissant grâce aux points de vue complémentaires qu'elles apportent sur un même objet d'étude. - L'article « Depositing Cult - Considerations on What Makes a Cult Deposit» (p. 25-48) de P. Pakkanen cherche à clarifier ce qu'on entend par "dépôts votifs" en redéfinissant dans un premier temps les termes « rituel » et «culte». L'A. s'applique ensuite à mettre en évidence en quoi les dépôts votifs renseignent sur le culte. Dans cette optique, elle estime qu'il est nécessaire d'étudier plus particulièrement la partie des dépôts qui peut être considérée comme des déchets ou comme des rebus. Puisque ces ensembles de matériel sont le résultat d'actes volontaires, ils peuvent directement être associés à la pratique cultuelle. P. Pakkanen introduit alors les bases d'une "archéologie des déchets» sous le néologisme de "garbology ". L'A. se risque aussi au comparatisme ethnologique en mettant en parallèle du matériel grec - celui mis au jour sur le sanctuaire de Poséidon à Kalaureia (île de Poros) - avec des objets issus de sites archéologiques précolombiens. - Cette première partie se termine avec l'article « Ash, Bones, Votives - Analysing the Black Strata in Early Greek Sanctuaries. Two Examples from Olympia - the Schwarze Schicht and the Altar of Artemis » (p. 9-64) de S. Bocher. L'A. y discute à nouveau des thématiques abordées dans les deux interventions précédentes mais traite plus particulièrement de la " couche noire » qu'on trouve de façon relativement récurrente sur différents sites archéologiques et qui constitue souvent le premier niveau de la séquence stratigraphique des sanctuaires antiques. Afin d'illustrer son propos, elle analyse deux exemples localisés sur le site d'olympie (ledit Pélopion et l'autel d'Artémis). En se référant à des études antérieures qui définissent un autel comme pouvant aussi être une accumulation de cendres, d'os d'animaux brûlés et d'objets votifs, S. Bocher soutient l'identification des "couches noires" comme les vestiges d'autels primitifs ou rudimentaires remontant aux origines des sanctuaires.

3 La seconde section, intitulée " Time and Material: Perspectives to Early Cult », regroupe quatre articles qui présentent des cas d'étude précis de sites archéologiques répartis sur une période allant du Bronze Récent aux premiers temps de l'époque archaïque. Dans «Private and Communal Ritual at Post-Palatial Tiryns » (p. 65-106), M. Vetters présente des statuettes en terre cuite datant du Bronze Récent mises au jour au sein de dépôts votifs sur le site de Tirynthe. L'A. remet alors en question la généralisation selon laquelle la présence de statuettes en terre cuite dans des dépôts implique qu'ils sont le résultat de pratiques cultuelles "publiques » et que leur absence dénote de pratiques cultuelles «domestiques". Après avoir déconstruit et rendu obsolète cette vision dichotomique, M. Vetters propose une catégorisation et une chronologie plus fines de ces terres cuites, d'une part en les mettant plus précisément en rapport avec le type de structure dans lesquelles elles ont été mises au jour, d'autre part en s'intéressant davantage à leur iconographie (figurines anthropomorphiques ou zoomorphiques). L'article « Interpreting Votive Offerings from Early Archaic Deposits at the Artemision of Ephesos» (p. 107-121) de G. Klebinder-Gau $\beta$ s'intéresse plus particulièrement aux offrandes métalliques provenant de deux dépôts votifs mis au jour dans l'enceinte de 
l'Artémision d'Éphèse, appartenant à la période géométrique et au début de l'époque archaïque. Après être revenue sur les problématiques méthodologiques et pratiques déjà longuement exposées dans les interventions précédentes, l'A. présente les spécificités du matériel d'Éphèse (nature, fonction et iconographie de la/des divinité[s] honorée[s]; formes, types et rôles des offrandes) puis les met brièvement en parallèle avec celles d'autres sanctuaires de la côte ionienne et du monde anatolien. - Dans "'Meeting with Others and the Gods': the Social Uses of Early Cretan Cult-Sites " (p.123-135), L. Sjörgen propose de considérer les espaces de culte non seulement comme des lieux où des dédicants apportent des offrandes aux divinités mais aussi comme des points de contacts et d'interactions entre les dédicants eux-mêmes, notamment à travers les offrandes plus prestigieuses. L'A. applique cette interprétation au matériel votif provenant de sanctuaires crétois remontant à l'âge du Fer et au début de l'époque archaïque. L. Sjörgen souligne ainsi la «compétition aristocratique » que laisse deviner ces offrandes et démontre alors l'interpénétration des pratiques rituelles et des comportements sociaux. - Le dernier article de cette seconde partie, «New Beginnings? Preparations of Renewal of Cult at Kalaureia and Asine » (p. 137-155) de B. Wells est édité à titre posthume par P. Pakkanen, A. Penttinen et J. Pakkanen. L'A. y commente trois dépôts situés dans le sanctuaire de Poséidon à Kalaureia (île de Poros) et un dépôt provenant du sanctuaire d'Asiné (Argolide), deux sites remontant au $\mathrm{VIII}^{\mathrm{e}}$ siècle dont elle dirigeait les fouilles. La particularité de ces quatre dépôts réside dans la quasi absence de matériel votif et dans leur relation avec des bâtiments sans fonction rituelle évidente, ce qui amène $\mathrm{B}$. Wells à considérer ces traces archéologiques comme les marqueurs de la réorganisation, de la transformation ou de la fondation voire de la refondation après destruction - de ces lieux de culte.

En fin de volume, onze planches illustrent en couleur certains des plans qui apparaissent déjà dans le corps du texte afin d'en permettre une lecture optimale. En conclusion, cet ouvrage souligne l'importance de la remise en contexte archéologique des dépôts votifs et met en garde contre l'interprétation de ce matériel en ayant recours à des grilles de lectures traditionnelles souvent biaisées. Il se propose alors de remettre en question et de réexaminer une série de problématiques liées à l'archéologie des lieux de culte et appelle de ses vœux la naissance d'une nouvelle vision de la pratique cultuelle dont il se veut le point de départ.

\section{AUTEURS}

GRAHAM CUVELIER

F.N.S.-FNRS - Université de Liège 